\title{
Silver-Palladium Nanodispersions in Silicate Matrices: Highly Uniform, Stable, Bimetallic Structures
}

\author{
Lawrence D’Souza, Parthasarathi Bera, ${ }^{*}$ and S. Sampath ${ }^{1}$ \\ Department of Inorganic and Physical Chemistry, ${ }^{*}$ Solid State and Structural Chemistry Unit, Indian Institute of Science, Bangalore-560 012, India
}

Received March 7, 2001; accepted October 22, 2001

\begin{abstract}
Ag-Pd nanobimetallic colloidal particles are prepared in a single step by a chemical reduction method. Organically modified aminosilicate is used as a supporting matrix as well as a stabilizing agent, to obtain very uniform, well-distributed bimetallic particles. These nanoparticles are found to be stable for several months in both the solid and the liquid phases. The structure of the bimetallic particles has been followed by X-ray photoelectron spectroscopy and ultraviolet-visible spectroscopy. The distribution and the particle size are determined by transmission electron microscope and $\mathrm{X}$-ray diffraction studies. Polymerization and condensation of the support silicate material have been confirmed by Fourier transform infrared spectroscopy. (c) 2002 Elsevier Science
\end{abstract}

Key Words: sol-gel; aminosilicate; nanoparticles; bimetal; Ag-Pd; $\mathrm{CO}$ adsorption.

\section{INTRODUCTION}

Research on nanoparticles has seen a sudden spurt over the last decade with emerging new areas like nanoelectronics and nanobiotechnology (1-12). The advantage in tuning many physical and chemical properties using a bimetallic combination has triggered special interest in the synthesis and stabilization of bimetallic particles over monometallic particles. Here, bimetal refers to particles containing two different kinds of metals, which has either a core-shell or an alloy structure and the kind of structure is decided by the method of preparation. Bimetals can be prepared by either physical or chemical routes. Physical routes mainly consist of vapor deposition of one metal on top of the other, whereas chemical methods involve simultaneous reduction of two metal ions or reduction of one after another in presence of a suitable stabilizer (13).

Catalysis is one of the extensively studied applications of nanoparticles. The catalytic property of transition and noble metals is greatly enhanced once the particles reach small sizes. Additionally, bimetals generate properties that are different from monometallic components (13). There are only a few reports on the synthesis and characterization of $\mathrm{Ag}-\mathrm{Pd}$ bimetallic particles. Venezia and co-workers synthesized pumice-supported Ag-Pd

\footnotetext{
${ }^{1}$ To whom correspondence should be addressed. Fax: 91-80-3601552. E-mail: sampath@ipc.iisc.ernet.in.
}

bimetallic catalysts and characterized them by X-ray diffraction (XRD) and X-ray photoelectron spectroscopy (XPS). The material was tested for the selective hydrogenation of diens (14). Huang and co-workers synthesized Ag-Pd bimetallic alloy particles by a chemical reduction method, characterized them by elemental analysis and XRD, and further used these particles for electronic component fabrication (15). Rao and co-workers synthesized fcc structured $\mathrm{Ag}-\mathrm{Pd}$ and $\mathrm{Cu}-\mathrm{Pd}$ nanoscale alloys in bulk quantities and characterized them with various techniques such as transmission electron microscopy (TEM) and XRD (16).

Recently, we have shown that Pt-Pd nanobimetallic particles having a core-shell structure can be prepared in a single-step process using silanes as stabilizers (17). In the present paper, we report on the preparation and characterization of Ag-Pd bimetallic particles using an organically modified silicate support matrix in solid and liquid phases. The silicate matrix acts as a stabilizer as well. The structure of the particles has been followed by various techniques such as XRD, XPS, CO adsorption and ultraviolet-visible (UV-Vis) spectroscopy.

\section{EXPERIMENTAL}

\section{Materials}

$N^{\prime}$-[3-(Trimethoxysilyl)propyl] diethylene triamine (TPDT) was obtained from Aldrich (U.S.A.). Hexachloroplatinate, palladium chloride, silver nitrate, sodium borohydride, methanol, and all other chemicals used were of analytical grade. Doubledistilled water was used.

\section{Apparatus}

UV-Vis spectra were recorded using a Hitachi 3000 spectrophotometer. The samples were in the form of sols. TEM was carried out using Model 3010 JEOL microscope operating at $300 \mathrm{kV}$. Samples were prepared by evaporating a drop of the sol on a copper grid. Polymerization proceeded during evaporation of the solvent and hence the TEM pictures reflect the distribution of metallic particles in the solid silicate samples. Enlarged photographs were used to determine the particle size. About 150 particles were considered for obtaining the histogram of the particle size distribution. Fourier transform infrared (FTIR) spectra were recorded using a Bruker IR Model Equinox 55 
spectrophotometer. EDX and SEM images of the solid silicate samples containing metallic particles were taken using a JEOL JSM-840A scanning microscope. Dried and powdered samples of the colloid with the stabilizer were used for the measurements. XRD analyses were performed using a Model XD-D1 Shimadzu diffractometer operating at $30 \mathrm{kV}$ and $30 \mathrm{~mA}$. CO adsorption measurements were carried out by purging the sols containing the desired metal particles followed by equilibration for about $30 \mathrm{~min}$. A few drops of the sol were placed on a $\mathrm{KBr}$ pellet and allowed to dry before the spectra was taken. XPS spectra were recorded on an ESCA-3 Mark II spectrometer (VG Scientific Ltd., England) using Al- $K \alpha$ radiation (1486.6 eV). Binding energies were calculated with respect to $\mathrm{C}(1 \mathrm{~s})$ at $285 \mathrm{eV}$. The analyses were performed on pellets of $8 \mathrm{~mm}$ diameter at a vacuum level of $10^{-9}$ Torr. Etching of the samples was carried out using $\mathrm{Ar}^{+}$ion sputtering at $6 \mathrm{kV}$.

\section{Preparation of Bimetallic Dispersions}

A typical preparation procedure for a bimetallic colloid containing 100:0.25 molar ratio of the precursor and the metal salts is as follows: $260 \mu \mathrm{L}$ of TPDT was added to $2 \mathrm{~mL}$ of methanol followed by $250 \mu \mathrm{L}$ of $\mathrm{H}_{2} \mathrm{O}$. This mixture was shaken for a few minutes, and $250 \mu \mathrm{L}$ each of $0.01 \mathrm{M} \mathrm{AgNO}_{3}$ and $0.01 \mathrm{M} \mathrm{PdCl}_{2}$ was subsequently added with vigorous stirring. The $\mathrm{AgCl}$ precipitate immediately dissolves in the silane to form Ag-silane complex. The resultant clear solution was diluted to $10 \mathrm{~mL}$ using methanol. The mixture was found to be very homogeneous and free from any precipitate. The $\mathrm{pH}$ of the solution was then adjusted to 5-6 using $0.1 \mathrm{M} \mathrm{HCl}$. Hydrolysis of the silance precursor was initiated during this period. After a few minutes, sodium borohydride $(0.005 \mathrm{~g})$ was added with vigorous stirring. The reduction reaction was monitored by measuring the UVvisible absorbance of the sol. Various compositions of the $\mathrm{Pd}$ and Ag, containing molar ratios of $1: 1,0.75: 0.25,0.5: 0.5$, and $0.25: 0.75$ of the salts, were prepared using the same protocol as described above. Control experiments were carried out with individual metal colloids and the physical mixture of previously formed individual metal colloids. In all the above preparations, no phase separation or segregation was observed. The color of bimetallic sols ranged from less intense brown to intense brown depending on the metal content. Colors of individual colloids were yellow for $\mathrm{Ag}$ and brown for Pd.

\section{RESULTS AND DISCUSSION}

The $-\mathrm{NH}_{2}$ group of the aminosilane is known to have good affinity toward noble metals and capable of stabilizing the metal particles (17-21). In the present case, the bimetal is prepared by simultaneous chemical reduction of two metal salts in the presence of a stabilizer that also acts as a support/matrix.

\section{UV-Visible Spectral Studies}

The characteristic surface plasmon absorption is a good tool for following the formation of nanoparticles and their reaction

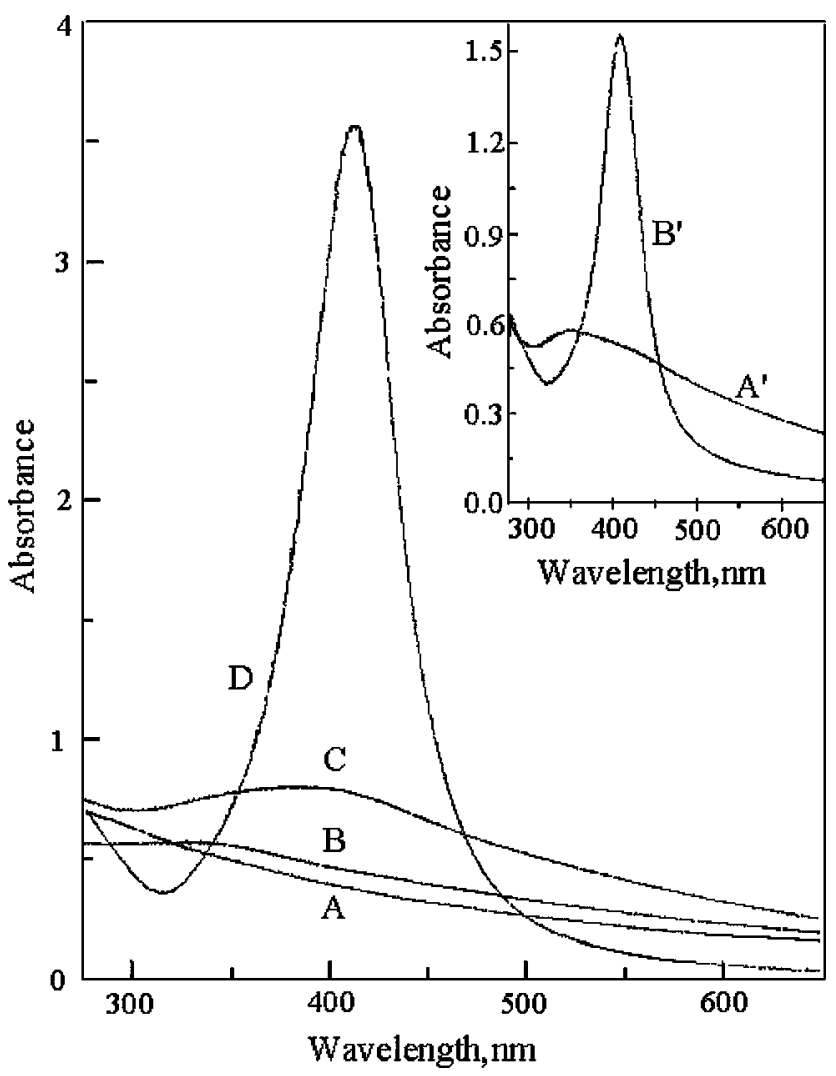

FIG. 1. Absorbance spectra of TPDT-stabilized Ag/Pd sol. (A) Pd, (D) Ag; (B) and (C) correspond to $\mathrm{Ag} / \mathrm{Pd}$ bimetallic colloids of molar ratios $0.75: 0.25$; $0.25: 0.75$ of the Pd and $\mathrm{Ag}$ salts, respectively. Inset: Absorbance spectra of TPDT-stabilized bimetallic colloid $\left(\mathrm{A}^{\prime}\right)$ and physical mixture of the same composition $\left(\mathrm{B}^{\prime}\right)$. The molar ratio of the silane to metal salts is $100: 0.5: 0.5$.

kinetics. Additionally, when following the simultaneous reduction of two metal systems, its advantage lies in knowing whether the resultant system is a bimetal, an alloy, or a physical mixture (22). It also gives information about the stability of the systems.

The monometallic dispersion of Pd is found to be very stable for several months without any aggregation or precipitation while that of Ag is not. This may be due to the low affinity of Ag towards the $-\mathrm{NH}_{2}$ group (18). However, bimetallic Ag-Pd particles show a good stability over a long period.

Figure 1 shows the surface plasmon absorption spectra of the sol containing different ratios of $\mathrm{Ag}$ and $\mathrm{Pd}$ bimetallic particles. The measurements are carried out after complete reduction of individual metal salts are ensured. $\mathrm{AgNO}_{3}$ does not absorb in the $\mathrm{UV}$-visible region, whereas $\mathrm{PdCl}_{2}$ gives two absorption peaks at 460 and $325 \mathrm{~nm}(17,20)$. The absence of these two peaks confirms the complete reduction of $\mathrm{PdCl}_{2}$. $\mathrm{Pd}$ particles give a broad absorption (Fig. 1A) over the entire visible range and is similar to what has been already reported (17-23). Nanosized silver particles absorb around $410 \mathrm{~nm}$, which is the characteristic surface plasmon peak. The absorption spectra for bimetallic particles of different molar ratios of Ag-Pd (0.75: 0.25, $0.5: 0.5,0.25: 0.75)$ are given in Figs. $1 \mathrm{C}, 1 \mathrm{~A}^{\prime}$, and $1 \mathrm{~B}$. The $\mathrm{Ag}$ plasmon band is 
greatly diminished and slightly blue-shifted once $\mathrm{Pd}^{2+}$ is also reduced simultaneously with $\mathrm{Ag}^{+}$. Similar observations have been reported by Torigoe and Esumi (24) for Ag-Pd bimetallic particles stabilized in poly ( $N$-vinyl-2-pyrrolidone). Figure $1 \mathrm{~B}^{\prime}$ is a physical mixture of $\mathrm{Ag}$ and $\mathrm{Pd}$ prepared from the individual colloids and the metal concentration is the same as that of the $0.5: 0.5$ bimetallic colloid $1 \mathrm{~A}^{\prime}$. The difference in spectral features in $1 \mathrm{~B}^{\prime}$ and $1 \mathrm{~A}^{\prime}$ is an additional proof for the bimetallic nature of the Ag-Pd particles. The change in the absorbance spectra of the bimetallic colloid compared to those of individual components can be primarily attributed to the change in dielectric function with different metal ions (25).

The structure or the core-shell nature of the bimetallic particles formed during simultaneous reduction of metal ions is mainly decided by the kinetics of reduction that in turn depends on the stability constant of the individual metal complexes. The stability constant of Pd-amine complex is much higher than that of the corresponding Ag-amine complex. The reported $\log K_{1}$ of the Pd-dien complex (26) is 34 while that of Ag-dien complex (27) is 6.1. Based on these values, it may be expected that the reduction process would result in the formation of $\mathrm{Ag}$ first and then Pd resulting in a core-shell type of structure with Ag as the core and Pd as the shell.

However, other studies such as XPS reveal the segregation of silver on the particle surface, as will be shown later. Secondly, $\mathrm{Pd}$ and $\mathrm{Ag}$ are known to form a series of solid solutions over the whole range of compositions (28). This might result in an alloy structure. Thirdly, the complete reduction of the Ag-dien complex to the corresponding metal takes place in $2.6 \mathrm{~min}$ while the Pd-dien complex takes $10 \mathrm{~min}$ for complete reduction under the present experimental conditions. This may give rise to the presence of different amounts of silver and palladium particles at any given time leading to the formation of an alloy since the two metals are miscible at any composition. Also, a compositional difference may be expected between the bulk and the surface. Indeed, this has been observed in the atomic ratios calculated from the XPS intensities.

The importance of $\mathrm{Si}-\mathrm{OH}$ and $-\mathrm{NH}_{2}$ groups has been studied and presented in our earlier work (17). It shows that the affinity of $-\mathrm{NH}_{2}$ toward metal, $\mathrm{Si}-\mathrm{O}-\mathrm{Si}$ network around the nanoparticles and the long alkyl chain together contributes to the stabilization of nanoparticles (17).

\section{XPS Studies}

XPS of the as-prepared Ag-Pd bimetallic sample does not show either Ag or Pd peaks. However the corresponding Ag or Pd peaks start to appear after 10 min of etching and the intensities of the $\mathrm{Ag}$ and $\mathrm{Pd}$ peaks increase with further etching of the sample. Binding energies of $\operatorname{Ag}\left(3 \mathrm{~d}_{5 / 2}\right)$ and $\mathrm{Pd}\left(3 \mathrm{~d}_{5 / 2}\right)$ at different etching times are given in Table 1 . The concentration ratios of $\mathrm{Ag}$ to $\mathrm{Pd}$ have been estimated using the following relation,

$$
\frac{C_{A g}}{C_{P d}}=\frac{I_{A g} \sigma_{P d} \lambda_{P d} D_{E}(P d)}{I_{P d} \sigma_{A g} \lambda_{A g} D_{E}(A g)},
$$

TABLE 1

Binding Energies $(\mathrm{eV})$ and Concentration Ratios $\left(C_{\mathrm{Ag}} / C_{\mathrm{Pd}}\right)$ of $\mathrm{Ag}$ and Pd at Different Times of Etching

\begin{tabular}{cccc}
\hline $\begin{array}{c}\text { Duration of etching } \\
(\mathrm{min})\end{array}$ & $\begin{array}{c}\text { Binding energy of } \\
\operatorname{Ag}\left(3 \mathrm{~d}_{5 / 2}\right)\end{array}$ & $\begin{array}{c}\text { Binding energy of } \\
\operatorname{Pd}\left(3 \mathrm{~d}_{5 / 2}\right)\end{array}$ & $C_{\mathrm{Ag}} / C_{\mathrm{Pd}}$ \\
\hline As prepared & - & - & - \\
10 & 368.1 & 337.9 & 3.2 \\
20 & 368.3 & 338.4 & 2.3 \\
30 & 368.0 & 338.0 & 1.3 \\
50 & 368.1 & 337.8 & 1.1 \\
\hline
\end{tabular}

where $C, I, \sigma, \lambda$, and $D_{E}$ are the concentration, intensity, photoionization cross section, mean escape depth, and geometric factor, respectively. Integrated intensities of $\mathrm{Ag}(3 \mathrm{~d})$ and $\mathrm{Pd}(3 \mathrm{~d})$ peaks have been taken into account in estimating the concentrations. Photoionization cross section and mean escape depths have been obtained from the literature $(29,30)$.

Both $\mathrm{Ag}$ and $\mathrm{Pd}$ peaks are observed after the first etching itself. The calculated concentration ratio of $\mathrm{Ag} / \mathrm{Pd}$ is 3.2 showing that there is a clear silver enrichment on the surface. Further etching reduces the ratio gradually and at an etching time of $30 \mathrm{~min}$ and above the ratio stays close to 1 . This confirms the fact that the particle consists of both silver and palladium at any stage. This rules out the possibility of a well-formed core-shell structure as observed for the Pt-Pd system using silane as the stabilizing agent (17).

The literature on the Ag-Pd alloy system clearly shows that the surface composition of Ag-Pd is very different from that of the bulk. For example, Noordermeer and co-workers (31) reported that the surface of a $\mathrm{Pd}_{0.67} \mathrm{Ag}_{0.33}$ alloy has a composition of $\mathrm{Pd}_{0.1} \mathrm{Ag}_{0.9}$. This enrichment of silver on the surface is explained based on the difference in surface energy of $\mathrm{Ag}$ and Pd. This is theoretically predicted as well, based on an ideal solution model and also based on a regular solution model taking the heat of mixing into account (32-34).

XPS of core level region of $\mathrm{Ag}(3 \mathrm{~d})$ and $\mathrm{Pd}(3 \mathrm{~d})$ after $30 \mathrm{~min}$ etching is given in Fig. 2. The $\mathrm{Ag}(3 \mathrm{~d})$ and $\mathrm{Pd}(3 \mathrm{~d})$ peaks in $\mathrm{Ag}-\mathrm{Pd}$ bimetallic cluster could be resolved into a set of respective spinorbit doublet. Accordingly, $\operatorname{Ag}\left(3 \mathrm{~d}_{5 / 2}\right.$ and $\left.3 \mathrm{~d}_{3 / 2}\right)$ doublet peaks are observed at 368.0 and $374.0 \mathrm{eV}$ (Fig. 2A) and they can be attributed to metallic $\mathrm{Ag}(35)$.

The binding energy values for palladium are observed at 338.0 and $343.1 \mathrm{eV}$ that correspond to $\operatorname{Pd}\left(3 \mathrm{~d}_{5 / 2}\right.$ and $\left.3 \mathrm{~d}_{3 / 2}\right)$ peaks (Fig. 2B). These values are shifted as compared to the bulk metal leading us to believe that the oxidation state of palladium may be different from zero. The binding energy value of $\operatorname{Pd}\left(3 \mathrm{~d}_{5 / 2}\right)$ has shifted by approximately $3 \mathrm{eV}$ as compared to the bulk metal $(335 \mathrm{eV})$. A careful examination of the literature reveals that $\operatorname{Pd}\left(3 \mathrm{~d}_{5 / 2}\right)$ in palladium oxide has a binding energy equal to $336.4 \mathrm{eV}$. Only ionic salts of palladium such as $\mathrm{PdCl}_{2}$ show a high shift of $3 \mathrm{eV}$ compared to the bulk metal (35). The formation of such salts can be ruled out in the present study. This is also confirmed by the XRD and UV-Vis studies that clearly 

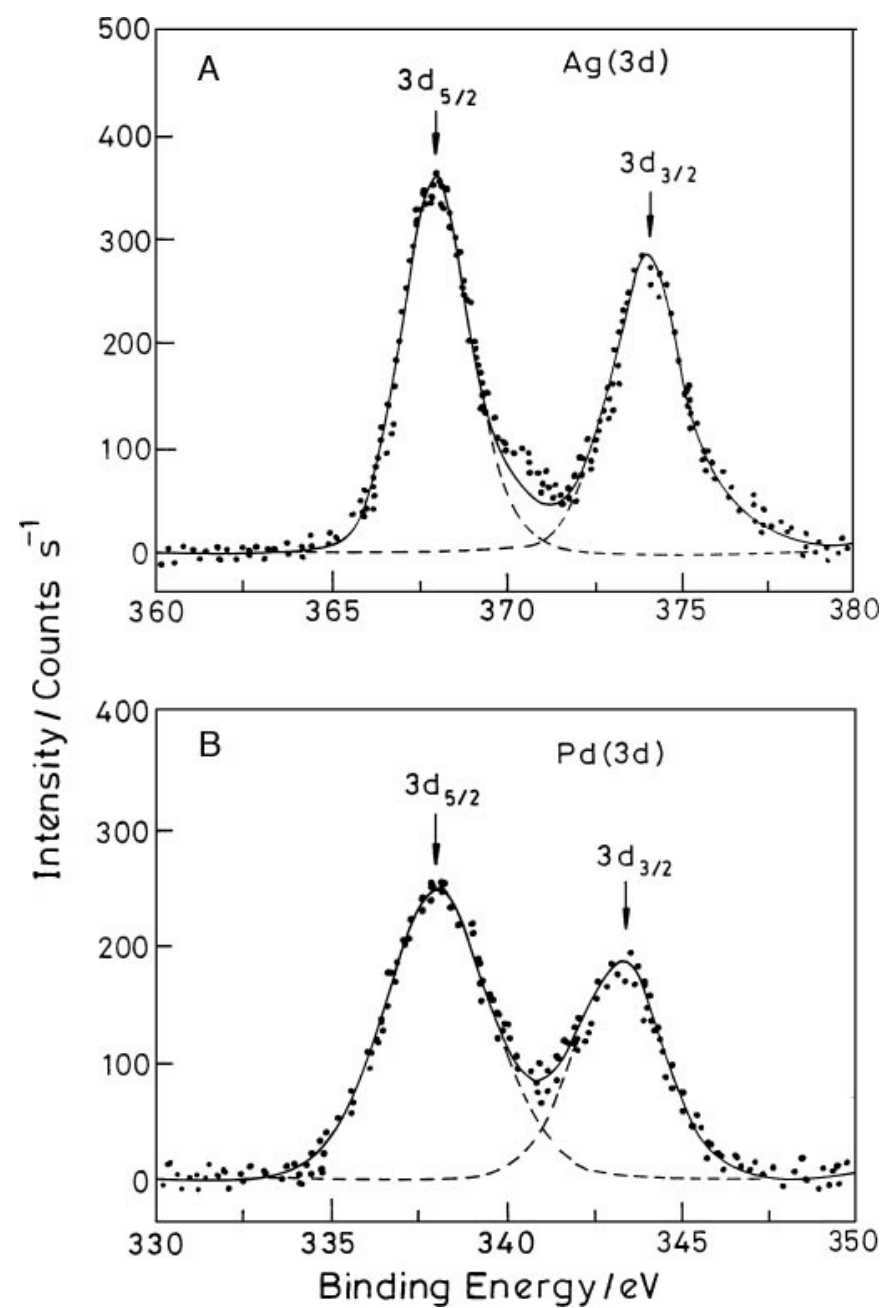

FIG. 2. XPS of core level regions of (A) $\mathrm{Ag}(3 \mathrm{~d})$ and (B) $\mathrm{Pd}(3 \mathrm{~d})$ in TPDTstabilized $\mathrm{Ag} / \mathrm{Pd}$ bimetallic colloid prepared from $\mathrm{Ag}$ and $\mathrm{Pd}$ metal salts of molar ratio $0.35: 0.65$ and etched for $30 \mathrm{~min}$.

show the formation of palladium metal. Secondly, the binding energy values do not shift with etching time (Table 1). Hence, there is no change (such as reduction of $\mathrm{Pd}^{2+}$ state) observed during argon ion etching.

The electronic structure aspects of surface metal layers and alloys have been reported for various systems including Ag-Pd. The core level binding energies of these metallic components are reported to shift considerably compared to bulk values due to charge transfer effects besides hybridization and final state effects $(36,37)$. The formation of metal-metal bonds causing a flow of electron density to the element with a larger fraction of empty states in the valence band at the initial stage will also contribute to the observed shift. Based on the valence band spectra, the appearance of a virtual bound state approximately $2.0 \mathrm{eV}$ below the Fermi level has also been documented (38). Another important factor is the formation of interfacial alloys that would also shift the core level binding energy. The shift is reported to depend on the fraction of palladium present in the particle (39). Additionally, the particle size being very small may also play a large role in this shift. Such a shift is not reported for Ag (39). Based on the above, we believe that both $\mathrm{Ag}$ and $\mathrm{Pd}$ are in their metallic state. The nature of the Ag-Pd particles prepared in the present study mainly consists of inhomogeneous alloy particles with a strong surface segregation of silver.

\section{TEM Studies}

Figure 3 shows the TEM pictures and the corresponding size distribution of the metal nanoparticles prepared from various concentrations of $\mathrm{AgNO}_{3}$ and $\mathrm{PdCl}_{2}$. The mean particle size slightly increases from $2 \mathrm{~nm}$ (Fig. 3A) to $2.5 \mathrm{~nm}$ (Fig. 3B) and further to $3 \mathrm{~nm}$ (Fig. 3C) with increasing concentrations of the metal salts with respect to silane (silane: $\mathrm{AgNO}_{3}: \mathrm{PdCl}_{2}$ ) from $100: 2.5: 2.5,100: 2.5: 3.75,100: 5: 7.5$, respectively. The standard deviation calculated based on the Figs. 3A, 3B, and $3 \mathrm{C}$ are $1.3(N-2=5), 1.1(N-2=5)$, and $1.8 \mathrm{~nm}$ $(N-2=10)$, respectively. The particle shape is nearly spherical in all the compositions and the size distribution is very narrow and unimodal. The absence of bimodal size distribution is also an indirect evidence of the formation of bimetal. A bimodal type of size distribution is generally expected due to the difference in growth rates for two metal colloids (25).

\section{XRD and SEM Studies}

The structure of the nanoparticles is difficult to determine from X-ray diffraction measurements especially when the particle size is very small and the reflections are broad. Figure 4 shows the powder XRD patterns of $\mathrm{Ag}, \mathrm{Pd}$, and Ag-Pd bimetal of composition $100: 5: 5$. The powder sample was heated to $300^{\circ} \mathrm{C}$ for $12 \mathrm{~h}$ to increase the crystallinity of the particles. $\mathrm{Ag}$ monometal shows peaks at $2 \theta$ values of 38.15 and 44.35 while Pd shows peaks at 39.52 and 45.48 corresponding to (111) and (200) planes. On the other hand, the bimetal shows reflections at 38.77 and 45.48 which lie in between the two monometals. Depending on the ratio of the two metals, the peaks shift toward either of the monometal reflections, whose concentration is high which is characteristic of an alloy $(13,19)$. Here, it is assumed that the nature of the particle does not change on heat treatment, and hence, this conclusion is to be taken with a certain degree of caution. The broadness of the peaks is due to the small size of the metal particles. The particle size of the bimetal is calculated using the Debye-Scherrer formula, $B=(0.9 \lambda) / t \cos \theta$ where $B$ is the broadening of diffraction line measured at half its maximum intensity, $\lambda$ is the wavelength of X-ray used, and $t$ is the diameter of the particle. The average particle size is calculated to be $3.2 \mathrm{~nm}$, which is slightly larger than that obtained from TEM measurements. The main difficulty in calculating the particle size from XRD measurements is the determination of breadth of the diffraction line. Simple measurements suffice to determine particle sizes up to $500 \AA$ accurately but good experimental techniques are required for size range beyond $500 \AA$ (40). The sharp peak and big particle size of the silver nanoparticle 

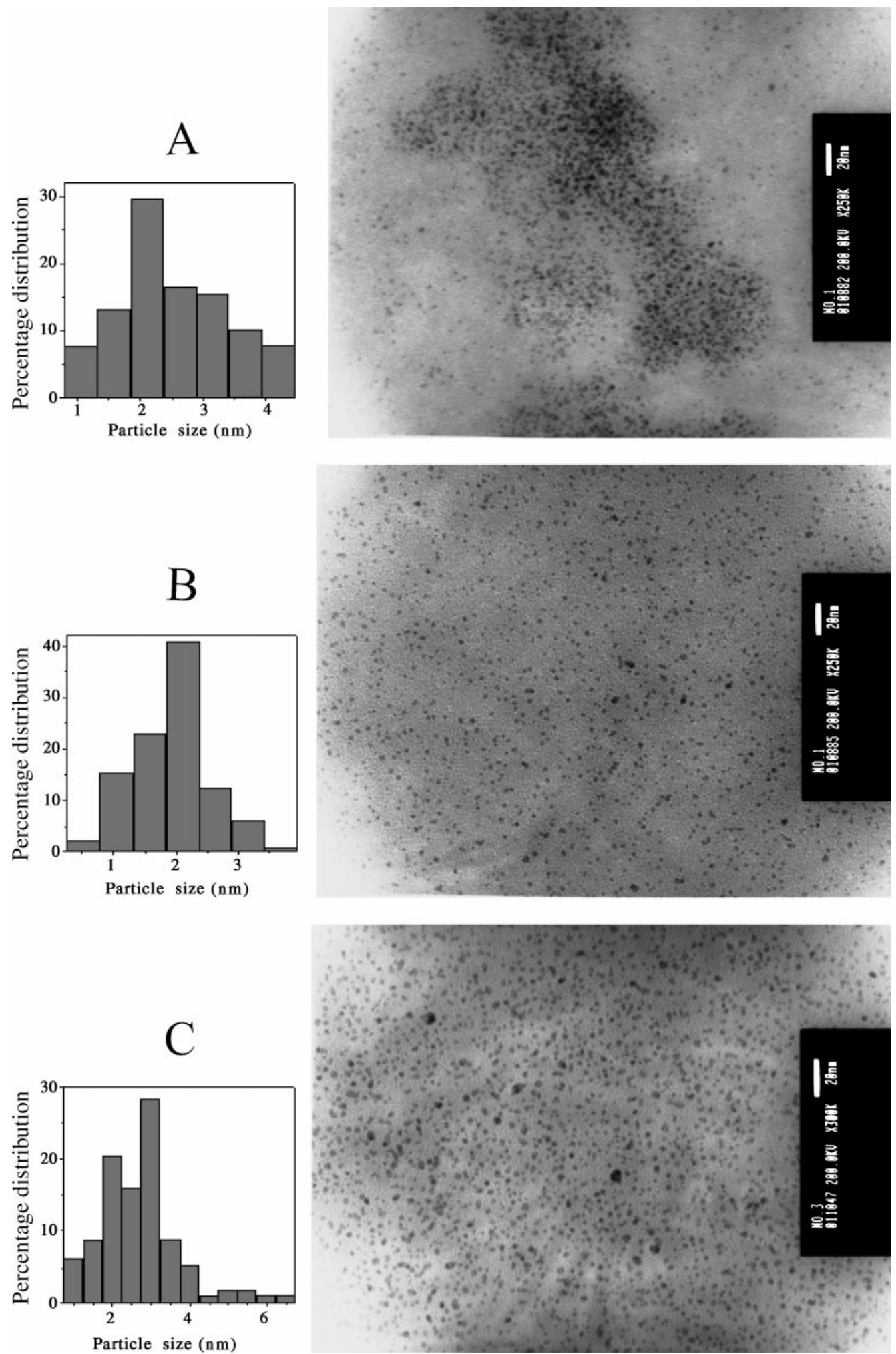

FIG. 3. TEM picture of the TPDT-stabilized Ag/Pd bimetallic colloid. The molar ratios of silane to Ag and Pd metal salts are (A) 100:2.5:2.5, (B) $100: 2.5: 3.75$, and (C) $100: 5: 7.5$, respectively. Bar indicates $20 \mathrm{~nm}$. The particle size distribution is also shown. 


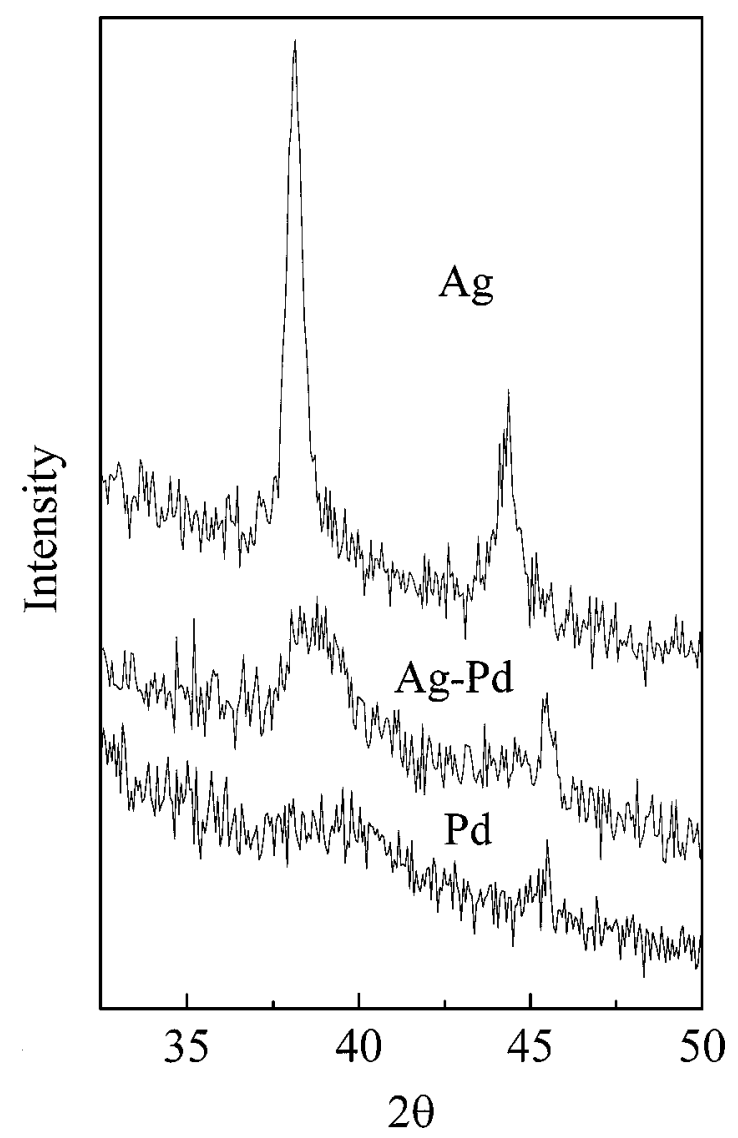

FIG. 4. XRD patterns of $\mathrm{Ag}, \mathrm{Pd}$, and bimetal of $\mathrm{Ag} / \mathrm{Pd}$. The molar ratios of silane to metal salts are 100:5 for Ag and Pd and 100:5:5 for bimetal.

is due to drastic growth of Ag by coalescence during the coagulation process (13).

Figure 5 shows the SEM pictures of the bimetal particles encapsulated in the silicate matrix. The corresponding elemental mapping clearly reveals that the metal particles are very uniformly distributed all over the matrix. The composition of the Ag-Pd salts used is in the molar ratio of $1: 3$. The morphology and particle size distribution remain the same for different compositions of the bimetal. Micropore formation in the silicate matrix was observed when the sol was evaporated and dried under warm $\left(50-70^{\circ} \mathrm{C}\right)$ conditions.

EDX was performed to determine the composition of the bimetals. Samples with different metal ratios were prepared and the solvent was evaporated to get dried samples. Samples were washed well with water and dried at $100^{\circ} \mathrm{C}$ for $10 \mathrm{~h}$ to get rid of chloride ions. The metal content obtained from EDX studies agrees well with the expected composition.

\section{CO Adsorption Studies}

$\mathrm{CO}$ adsorption on metals is a well-known phenomenon and is frequently used in the literature $(17,19,41,42)$ for obtaining the structure of bimetallics. Many metals like Pd and $\mathrm{Pt}$ adsorb CO strongly at NTP, but adsorption of $\mathrm{CO}$ on $\mathrm{Cu}$,
$\mathrm{Au}$, and $\mathrm{Ag}$ is by contrast very weak and largely physical in nature (43). Bradshaw and Pritchard could observed the $\mathrm{CO}$ adsorbed on $\mathrm{Ag}$ at very low temperatures of $113 \mathrm{~K}$ and high pressures of $\mathrm{CO}$ of $1 \times 10^{-1} \mathrm{~N} \mathrm{~m}^{-2}$ by FTIR (43).


FIG. 5. SEM picture (A) of TPDT matrix containing 2.5 : 7.5 molar ratio of $\mathrm{Ag}$ and $\mathrm{Pd}$. The elemental mapping is also shown: (B) $\mathrm{Ag}$ and (C) $\mathrm{Pd}$. 
The present studies are carried out at ambient temperature and pressure. No adsorption peak was observed for $\mathrm{CO}$ on Ag colloid (Fig. 6A). However, two strong peaks at 2067 and $1903 \mathrm{~cm}^{-1}$ (Fig. 6E) are observed for a Pd sample that correspond to $\mathrm{CO}$ adsorbed on $\mathrm{Pd}$ at the terminal site and the bridging site, respectively. In the case of bimetals, two peaks are observed close to 2065 and $1900 \mathrm{~cm}^{-1}$, depending on the composition. With increasing concentration of $\mathrm{Ag}$, these two peaks shift slightly toward lower the frequency region with decreasing intensity. For the bimetal of molar composition $\mathrm{Ag} / \mathrm{Pd}=$ $1: 3$ (Fig. 6D) peaks appear at 2065 and $1878 \mathrm{~cm}^{-1}$, for $\mathrm{Ag} / \mathrm{Pd}=$ $1: 2$ (Fig. 6C) at 2065 and $1876 \mathrm{~cm}^{-1}$, and for $\mathrm{Ag} / \mathrm{Pd}=1: 1$, (Fig. 6B) at 2061 and $1866 \mathrm{~cm}^{-1}$. Only palladium peaks are observed at any composition, thus revealing that the adsorption on the exposed silver is not strong.

\section{FTIR Spectral Studies}

Hydrolysis and polymerization of the support silicate network are followed by infrared spectral studies. Figure 7A shows FTIR spectrum for neat TPDT and 7B for the bimetal encapsulated, polymerized TPDT. Completely polymerized and dried gel is used in the latter case. The absence or presence of very weak bands that are characteristic of methoxy groups (at 1190, 1056, and $2880 \mathrm{~cm}^{-1}$ ) in the metal stabilized silicate sample indicates

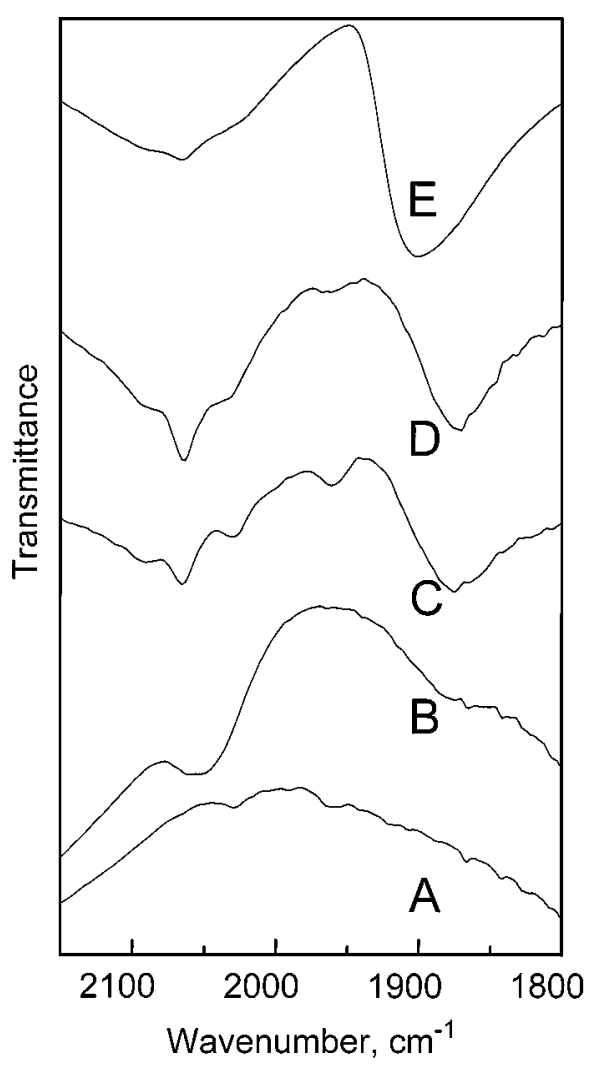

FIG. 6. FTIR spectra of CO adsorbed on the TPDT-stabilized metallic particles. (A) Ag, (E) Pd; Ag/Pd bimetal of molar ratio (B) $1: 1$, (C) $1: 2$, and (D) $1: 3$.

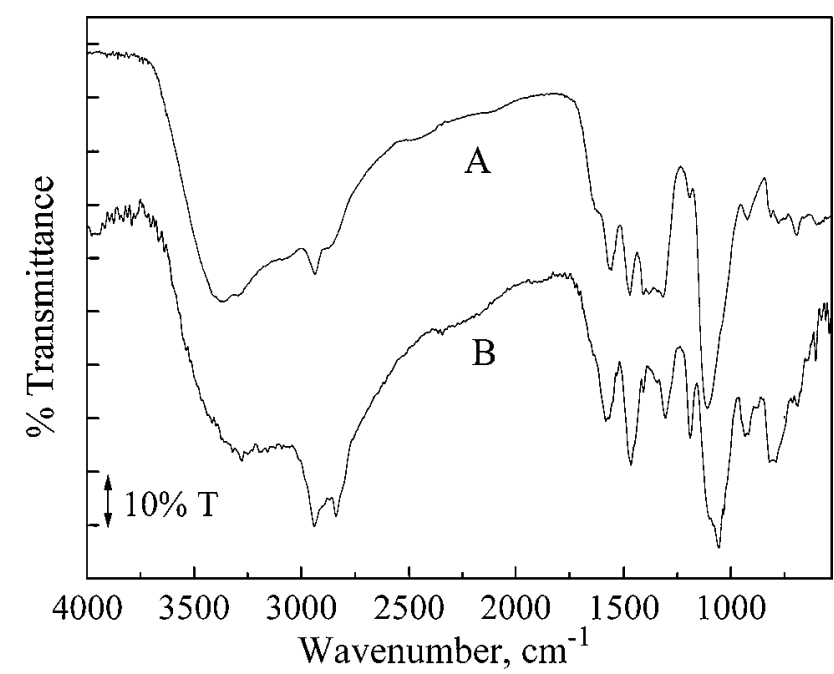

FIG. 7. FTIR spectra of TPDT-stabilized (A) bimetallic colloid of composition $0.5: 0.5$, and (B) corresponds to that of neat TPDT.

that the polymerization is nearly complete. The presence of a predominant peak at $1110 \mathrm{~cm}^{-1}$ is characteristic of the formation of a siloxane network. The low intensity of the band at $\sim 925 \mathrm{~cm}^{-1}$ indicates that the remaining unaffected $\mathrm{Si}-\mathrm{O}$ of the surface silanol groups is very low. The high intensity of the siloxane band at around $1100 \mathrm{~cm}^{-1}$ indicates the presence of linear siloxane chains in the network. It is already reported by Buining and co-workers (44) that aminosilane coating of mercaptosilanemodified gold particles exhibited a sharp band at $1113 \mathrm{~cm}^{-1}$ very similar to that of linear dimethylsiloxane. This observation was attributed to the formation of linear networks. Hence, the present material is likely to be made of linear siloxane chains around the $\mathrm{Ag} / \mathrm{Pd}$ alloy nanoparticles as was pictorially visualized by Bharathi and co-workers for gold nanoparticles (18).

\section{CONCLUSIONS}

Ag-Pd nanobimetallic particles are prepared by simultaneous reduction of the corresponding salts with $\mathrm{NaBH}_{4}$ in a single step using aminosilicate as a stabilizer and support. Bimetallic particle formation is confirmed by UV-visible spectroscopy. XPS studies reveal that the structure consists of inhomogeneous alloy clusters with silver surface segregation. XRD studies carried out with heat-treated samples confirm the nature of alloy clusters. TEM studies reveal that the average particle size is around $2 \mathrm{~nm}$. The metal distribution is found to be very uniform based on SEM and elemental analysis.

\section{ACKNOWLEDGMENTS}

The authors thank Professor M. S. Hegde and V. Jayaram, Solid State and Structural Chemistry Unit, Indian Institute of Science, for the XPS studies. Financial support from the Department of Science and Technology (DST), Government of India, and Council of Scientific and Industrial Research (CSIR), New Delhi, is gratefully acknowledged. 


\section{REFERENCES}

1. Lowe, C. R., Curr. Opin. Struct. Biol. 10, 428 (2000).

2. Lava, J.-M., Thomas, D., and Mazeran, P.-E., Analyst 125, 29 (2000).

3. Schmid, G., and Lifeng, F. C., Adv. Mater. 10, 515 (1998).

4. Dagata, J. A., Nanotechnology 8, 3 (1997).

5. Bandyopadhyay, S., Phys. Rev. B 61, 13813 (2000).

6. Prost, W., Kruis, F. E., Otten, F., Nielsch, K., Rellinghaus, B., Auer, U., Peled, A., Wassermann, E. F., Fissan, H., and Tegude, F., J. Microelectron. Eng. 41/42, 535 (1998).

7. Driskill-Smith, A. A. G., Hasko, D. G., and Ahmed, H., Microelectron. Eng. 53, 179 (2000).

8. Rossum, M. V., Mater. Sci. Eng. B 20, 128 (1993).

9. Baron, T., Martin, F., Mur, P., Wyon, C., Dupuy, M., Busseret, C., Souifi, A., and Guillot, G., Appl. Surf. Sci. 164, 29 (2000).

10. Baron, T., Martin, F., Mur, P., Wyon, C., and Dupuy, M., J. Cryst. Growth 209, 1004 (2000).

11. Zacarias, A. G., Castro, M., Tour, J. M., and Seminario, J. M., J. Phys. Chem. A 103, 7692 (1999).

12. Pum, D., and Sleytr, U. B., Trends Biotechnol. 17, 8 (1999).

13. Toshima, N., and Yonezawa, T., New J. Chem. 1179 (1998).

14. Venezia, A. M., Liotta, L. F., Deganello, G., Schay, Z., and Guczi, L., J. Catal. 182, 449 (1999).

15. Huang, C.-Y., Chiang, H.-J., Huang, J.-C., and Sheen, S.-R., Nanostruct. Mater. 10, 1393 (1999).

16. Vasan, H. N., and Rao, C. N. R., J. Mater. Chem. 5, 1755 (1995).

17. D'Souza, L., and Sampath, S., Langmuir 16, 8510 (2000).

18. Bharathi, S., Fishelson, N., and Lev, O., Langmuir 15, 1929 (1999).

19. Toshima, N., and Wang, Y., Chem. Lett., 1611 (1993); Toshima, N., Harada, M., Yonezawa, T., Kushihashi, K., and Asakura, K., J. Phys. Chem. 95, 7448 (1991).

20. Yonezawa, T., and Toshima, N., J. Chem. Soc., Faraday Trans. 91, 4111 (1995).

21. Liz-Marzán, L. M., Giersig, M., and Mulvaney, P., Langmuir 12, 4329 (1996).

22. Mulvaney, P., Langmuir 12, 788 (1996).
23. Esumi, K., Shiratori, M., Ishizuka, H., Tano, T., Torigoe, K., and Meguro, K., Langmuir 7, 457 (1991); Miner, R. S., Jr., Namba, S., and Turkevich, J., "Proceedings of the Seventh International Congress on Catalysis" (T. Seiyama and K. Kodansha, Eds.), p. 160. Tokyo, 1981.

24. Torigoe, K., and Esumi, K., Langmuir 9, 1664 (1993).

25. Torigoe, K., Nakajima, Y., and Esumi, K., J. Phys. Chem. 97, 8304 (1993).

26. Stability constants of metal-ion complexes, Part B, IUPAC Chemical Data Series No. 22, compiled by D. D. Perrin.

27. Stability constants of metal-ion complexes, Special Publication No. 17, The Chemical Society, compiled by L. G. Sillen and A. E. Martell, 1964.

28. Kuijers, F. J., and Ponec, V., J. Catal. 60, 100 (1979).

29. Scofield, J. H., J. Electron Spectrosc. Relat. Phenom. 8, 129 (1976).

30. Penn, D. R., J. Electron Spectrosc. Relat. Phenom. 9, 29 (1976).

31. Noordermeer, A., Kok, G. A., and Nieuwenhuys, B. E., Surf. Sci. 165, 375 (1986).

32. Williams, F. L., and Nason, D., Surf. Sci. 45, 377 (1974).

33. Van Santen, R. A., and Boersma, M. A. M., J. Catal. 34, 13 (1974).

34. Tyson, W. R., and Miller, W. A., Surf. Sci. 62, 267 (1977).

35. Briggs, D., and Seah, M. P., "Practical Surface Analysis by Auger and X-Ray Photoelectron Spectroscopy," Appendix 4. Wiley, New York, 1984.

36. Santra, A. K., and Rao, C. N. R., J. Phys. Chem. 98, 5962 (1994).

37. Wertheim, G. K., and Rowe, J. E., Science 260, 1527 (1993).

38. Hüfner, S., Wertheim, G. K., and Wernick, J. H., Phys. Rev. B 8, 4511 (1973).

39. Santra, A. K., and Rao, C. N. R., Appl. Surf. Sci. 84, 347 (1995).

40. Cullity, B. D., "Elements of X-ray Diffraction," Addison-Wesley, Reading, MA, 1967.

41. Wang, Y., and Toshima, N., J. Phys. Chem. B 101, 5301 (1997).

42. Bradley, J. S., in "Clusters and Colloids" (G. Schmid, Ed.), p. 510. Weinheim, Germany, 1994.

43. Bradshaw, A. M., and Pritchard, J., Proc. Roy. Soc. London A 316, 169 (1970).

44. Buining, P. A., Humbel, B. M., Philipse, A. P., and Verkleij, A. J., Langmuir 13, 3921 (1997). 